\title{
SIMULATION BRANCHING PROCESSES BY MIXING DISTRIBUTIONS
}

\author{
BEHROUZ FATHI VAJARGAH \\ and
}

MOJTABA MORADI

\begin{abstract}
The main aim of this paper is to study the behavior of mixed distributions in branching processes. We show that the mixed distribution cannot effect on the number of particles in the $n^{\text {th }}$ period and it must be ignored for further simulations.
\end{abstract}

Mathematics Subject Classification 2000: 60J80, 65C40, 65C05

Additional Key Words and Phrases: Branching processes, Monte Carlo, Mixed distributions.

\section{INTRODUCTION}

One of the first applicable models of the complex dynamical systems arose from studies of stochastic branching processes in the development of families and populations. A branching process model describes a system of randomly proliferating particles. The particles considered might be whole organisms, cells, molecules, individual, or any other set of objects which are organized into lineage of reproducing individuals. The particles in a branching process live for a time and have offspring according to a prescribed probability distribution. There are many variants of branching process models. The more famous models are called Galton Watson (GW) processes. The GW model describes a papulation where particles live for exactly one unit of time (a generation), and at the moment of death it produces a random number of progeny according to a prescribed probability distribution. Each of the first-generation progeny behaves independent of each other, and acts as the initial particle. It lives for a unit of time and produces a random number of progeny. Each of the second-generation progeny behaves in the identical way, and so forth. From the fact that the life spans of all particles are identical and equal to 1 , it follows that the process can be mathematically described using a discrete-time index, identical to the number of successive generation.[1]

In the theory of branching processes, GW branching process is a sequence of random variables $Z_{n}, n=0,1,2, \ldots$ defined by

$$
Z_{n+1}=\sum_{i=1}^{Z_{n}} \xi_{i} \quad, \quad Z_{0}=1
$$

where $\xi_{i}$ are independent, non-negative integer valued and identically distributed 


\section{BEHROUZ FATHI VAJARGAHandMOJTABA MORADI}

random variables with distribution $p_{k}, k=0,1,2, \ldots\left(P\left(\xi_{i}=k\right)=p_{k}\right) . \quad p_{k}$ is so called offspring distribution or reproduction law. $Z_{n}$ denotes the size of the population in the $n^{\text {th }}$ generation and condition $Z_{0}=1$ means that the process starts with just one particle. The reproduction mean or the mean number of offspring is defined by $m=E\left(\xi_{i}\right)=\sum_{k=0}^{\infty} k p_{k}$. The expected size of the $n^{t h}$ generation satisfies

$$
E\left(Z_{n}\right)=m E\left(Z_{n-1}\right)=m^{n} E\left(Z_{0}\right) .
$$

A generalization of branching processes is when the distribution of $\xi_{i}$ varies across individuals. For example, let $\theta$ be a random variable and also the conditional random variable $\xi \mid \theta$ has poisson distribution, which is called mixing distribution. A certain mixture distribution arises when all (or some) parameters of a distribution vary according to some probability distribution. Mixed distributions for branching processes, usually use in many branches of biology and epidemiology applications. For more details, see $[1 ; 2]$. The main aim of this paper is to compare the behavior of various types of the mixed distributions in the GW branching process. In fact, by Monte Carlo simulation, we want to show wether the mixed distributions in branching processes, are ineffective?

Suppose that offspring distribution follow as the mixed distribution of $\xi \mid \theta$, where $\theta$ is a random variable and $\xi \mid \theta$ has poisson distribution. We choose a situation that $E(\theta)$ be the same for all desired distributions. Then we study the behavior of $Z_{n}$ by the Monte Carlo simulation, when a particle generates new particles according to poisson distribution with parameter $\theta$.

\section{MONTE CARLO ALGORITHM AND SIMULATION}

In this section, using the Monte Carlo simulation, we approximate the number of particles in $n^{\text {th }}$ generation, means the value of $Z_{n}$. First, we obtain the value of $\theta$ by generating a random number based on a given distribution and then we generate a random number according to a poisson distribution with parameter $\theta$, as a number of offspring for a single particle. Monte Carlo algorithm for simulation of $Z_{n}$ is as follow:

\section{Monte Carlo Algorithm:}

(1) Get the variable $m$ as the number of Markov chains (the number of interactions of the algorithm).

(2) Get the value of $n$.

(3) Get the number of particles in zero generation $\left(Z_{0}\right)$.

(4) Get the distribution of random variable $\theta$ and set it as $g(\theta)$.

(5) Set $k=1$.

(6) Set $i=1$.

(7) Set $Z_{i}=0$.

(8) Set $j=1$.

(9) Generate $\hat{\theta}$ according to $g(\theta)$.

(10) Generate $\xi$ according to poisson distribution with the parameter $\hat{\theta}$.

(11) Set $Z_{i}=Z_{i}+\xi$. 
(12) Set $j=j+1$, if $j \leq Z_{i-1}$ goto step 9 .

(13) Set $i=i+1$, if $i \leq n$ go to step 7 .

(14) Set $s u m=s u m+Z_{i}, k=k+1$, and if $k \leq m$ go to step 6 .

(15) Obtain sum $/ k$ as Monte Carlo simulation for $Z_{n}$.

Computational results for variate distributions of $\theta$, illustrated in table 1 . Results in this table obtained for $Z_{0}=5, n=10$, so we have $E\left(Z_{n}\right)=2500$. The number of employed Markov chains are 1000.

Table 1: Monte Carlo estimation of $Z_{n}$, where a particle generates its offspring according to a Poisson distribution whose parameter is determined by $g(\theta)$ distribution, such that $E(\theta)=2$ and $E\left(Z_{n}\right)=2500$.

\begin{tabular}{|c|c|c|}
\hline distribution of $g(\theta)$ & parameter(s) & Monte Carlo estimation \\
\hline \hline Poisson & $\mathrm{P}(2)$ & 2580.9 \\
Uniform & $u(1,3)$ & 2644.2 \\
Binomial & $\mathrm{B}(5, .4)$ & 2486.5 \\
Geometric & $\mathrm{G}(0.5)$ & 2532.6 \\
Exponential & $\exp (2)$ & 2431.7 \\
\hline
\end{tabular}

The obtained results in table 1 show that the number of generated particles are independent of mixed distributions. In this table, we use different mixed distributions in such a way that the reproduction number be equal to 2 . Reproduction number, $R_{0}$, is the expected number of particles produced by an particle throughout its life. Under general conditions, if $R_{0} \leq 1$, the branching process becomes extinct with probability one. But, if $R_{0}>1$, there still may be a positive probability, say $q$, that the branching process becomes extinct.

\section{CONCLUSION}

In this paper, using Monte Carlo simulation based on obtained results in table 1 , we showed that the total particles in $n^{\text {th }}$ generation are independent of mixed distributions. Since using mixed distributions cannot effect on the size of $Z_{n}$, then for future works based on above mixed distributions in simulating branching processes are ineffective and useless. 


\section{BEHROUZ FATHI VAJARGAHandMOJTABA MORADI}

\section{REFERENCES}

[1] F. Brauer , P. v. d. Driessche, J. Wu (Eds.), Mathematical Epidemiology, Springer, 2008.

[2] J. O. Lloyd-Smith, S. J. Schreiber, P. E. Kopp , W. M. Getz, Superspreading and the effect of individual variation on disease emergence,LETTERS, Vol 438(17), 2005.

[3] P. Guttorp, Statistical Inference for Branching Processes, Wiley Ser. Probab. Math. Statist., Academic Press, New York, 1991.

T.E. Harris, The Theory of Branching Processes, Springer-Verlag, Berlin, 1963.

[4] M. Kimmel, E.A. David, Branching processes in biology, Springer-Verlag, New York, 2002.

[5] M. Mendoza, GE. utirrez-Pea, Bayesian conjugate analysis of the GaltonWatson process. Test 9 (1), 149-171. 2000.

[6] T.N. Sriram, A. Bhattacharya, M. Gonzlez, R. Martnez, I. del Puerto, Estimation of the offspring mean in a controlled branching process with a random control function, Stochastic Processes and their Applications, 117 : 928-946(2007).

\section{BEHROUZ FATHI VAJARGAH}

and

MOJTABA MORADI

Department of Statistics,

Faculty of Mathematical Sciences,

University of Guilan, Rasht,

Iran.

email: fathi@guilan.ac.ir; mmoradi@guilan.ac.ir 